\title{
0 uso de simulação como metodologia de pesquisa em ciências sociais
}

Paulo Vicente*

\section{Resumo}

A simulação é apresentada como uma metodologia pouco utilizada nas ciências sociais, quando comparada ao estudo de caso e à comparação, como forma de superar a dificuldade de realização de experimentos.

A simulação é um "experimento virtual" que requer um modelo operatório representando no todo (ou em parte) um sistema ou processos que o caracterizem. 0 uso dessa metodologia é analisado como forma de buscar um modelo, confirmá-lo ou fazer uma projeção de eventos futuros. Dessa forma, a simulação se presta tanto no "contexto de descoberta" quanto no "contexto da prova". Cada uma dessas três formas de uso da metodologia é discutida, bem como as condições nas quais devem ser feitas simulações e quais suas vantagens e limitações.

0 artigo observa que devido ao aperfeiçoamento dos computadores e de seus programas nas duas últimas décadas, a simulação teve sua utilização muito simplificada. 0 texto ainda apresenta dois exemplos interativos utilizando o Excell, um sobre teoria da complexidade aplicada ao marketing e outro sobre teoria microeconômica.

Palavras-chave: simulação, pesquisa, computadores, metodologia

\section{Abstract}

Simulations are presented as a methodology that has been neglected in use by the social sciences when compared to the case study and the comparison, as a way to overcome the difficulty to run experiments. The simulation is a "virtual experiment", that requires an operating model representing the system, or processes that characterize it, as a whole or part. The use of this methodology is studied as a form of searching a model, confirming a model or making a projection about future events; in this way it serves both the "context of discovery" and the "context of proof" in sciences. Each of these uses is discussed, as well as the conditions in which simulations should be used and its advantages and limitations. The article explains that due to the advance of computers and their softwares in the last two decades this resource became much simpler to use, and two interactive examples using Excell are introduced, one about complexity theory applied to marketing and the other about microeconomic theory.

Keywords: simulation, research, computers, methodology

\section{Introdução}

Uma das maiores dificuldades das ciências sociais é a da experimentação, o que também se reflete na administração. $\mathrm{O}$ ambiente no qual as organizações estão inseridas se altera constantemente, seja pela adoção de novas tecnologias, ações competitivas dos concorrentes, decisões do governo em todos os níveis (federal, estadual e municipal) ou por mudanças no comportamento dos indivíduos, tanto dentro quanto fora da organização. Por isso, é muito difícil realizar experimentos como nas ciências naturais, onde os fatores são mais controláveis e podem ser alterados, um por um, analisando-se isoladamente o impacto que causam. No mundo organizacional, vários fatores se alteram ao mesmo tempo independentemente da vontade do pesquisador.

Bruyne (1977) classifica quatro modos de investigação, conforme mostrado no quadro 1: estudos de caso, comparação, experimentação e simulação. Embora sob certas condições controladas seja possível fazer experimentações, a dificuldade de lidar com estas e controlar as condições tem levado a administração a se

"Professor do Núcleo de Pesquisa da ESPM. Doutorando da EBAPE.

Artigo recebido em novembro de 2004 e aceito em dezembro de 2004. 
concentrar em estudos de caso e comparações. Entretanto, desses modos de investigação, a simulação é o que tem sido relativamente negligenciado.

Kleiboer (1997) indica cinco funções para as simulações: ferramenta de pesquisa, instrumento de ensino, método de planejamento, ferramenta de suporte à decisão e método de seleção de pessoal; sendo que este estudo se concentra apenas na função "ferramenta para pesquisa". O objetivo é auxiliar um pesquisador das ciências sociais - especialmente da administração - a entender como pode se valer dos avanços em computação verificados nos últimos anos para avançar em sua linha de pesquisa. Ainda que nos últimos anos softwares de utilização cada vez mais simplificada tenham sido lançados no mercado, as ciências sociais tem sido lentas em assimilar essa nova tecnologia. Embora o propósito deste artigo não seja entender o motivo pelo qual as simulações são negligenciadas, acredita-se que exista a barreira do conhecimento sobre programação em algumas áreas da administração.

De acordo com Bruyne (1977), a simulação tem a vantagem de ocorrer num ambiente controlado e fechado. Esta tem igualmente a vantagem de poder levar em consideração uma grande quantidade de variáveis ao mesmo tempo. Mesmo interagindo de forma simples, o comportamento conjunto pode se tornar complexo (STERMAN, 2001), o que limitaria o entendimento do conjunto devido à incapacidade do cérebro humano de lidar simultaneamente com um número crescente de variáveis, devido à racionalidade limitada (DOYLE, 1999).

Quadro 1

Modos de investigação

\begin{tabular}{|c|c|c|c|}
\hline ESTUDO DE CASO & COMPARAÇÃO & EXPERIMENTAÇÃO & SIMULAÇÃO \\
\hline Real & & & Artificial \\
\hline Aberto & & & Fechado \\
\hline Descontrolado & & & Controlado \\
\hline
\end{tabular}

Fonte: Bruyne (1977, p.223)

Como desvantagens principais, as simulações têm a limitação de gerar fenômenos artificiais e necessitar de um modelo operacional anterior, no qual as variáveis interagem. Assim, as variáveis precisam ser operacionalizadas de alguma forma, mesmo simplificada, do tipo "verdadeiro ou falso" ou "presente ou nãopresente".

\section{Usos da simulação}

Inicialmente, é preciso definir o que se entende por simulação. Em Bruyne (1977), a simulação é entendida como "a construção e a manipulação de um modelo operatório representando todo, ou parte de, um sistema ou processos que o caracterizam". Greenblatt (apud KLEIBOER, 1997) define simulação como um "modelo refletindo as características centrais de um sistema, processo ou ambiente, real ou proposto".

É importante observar que levando em conta essas definições não é preciso um computador, pois é possível fazer uma simulação apenas por conjecturas do tipo "e se", que podem ser mais abertas e chegar mais perto de técnicas de cenários (HEIJDEN, 1996; RINGLAND, 1998). Kleiboer (1997) coloca a técnica de cenários na mesma categoria de simulações, sendo dois conceitos diferentes sobre como modelar o mundo e aprender interativamente. Reibstein e Chussil (1999) colocam tanto as simulações quanto os cenários dentro da categoria "técnicas de predição".

O uso de modelos está mais associado ao paradigma funcionalista de Burrel e Morgan (1982), tanto dentro do que esses autores classificam como "objetivismo" quanto dentro das "disfunções burocráticas". Isso significa que todas as linhas de pesquisa que utilizam esse paradigma e se valem de funções e modelos dentro da teoria dos sistemas podem se valer desse tipo de metodologia de pesquisa. Na administração isso inclui marketing, logística, finanças, produção e estratégia, tanto na área da gestão pública quanto na de empresas privadas. 


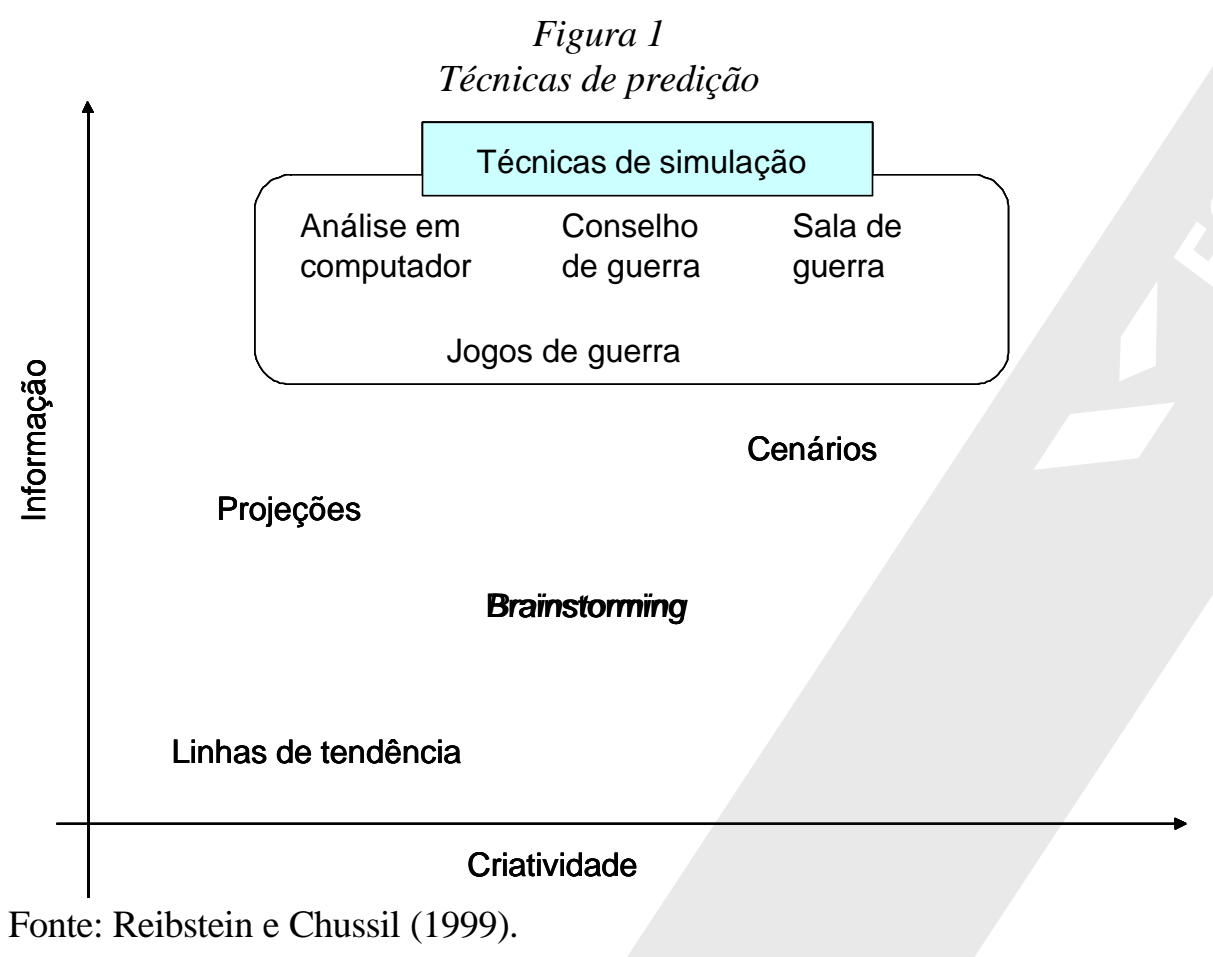

É comum achar que os recursos humanos não podem se valer desse tipo de metodologia. Entretanto, como as pessoas têm perfis psicológicos que não se alteram, sua reação numa simulação é muito similar à da vida real. Isso significa, por exemplo, que é possível observar suas reações ao risco e a situações de conflito dentro de uma simulação. Pode-se, contudo, categorizar o uso das simulações a partir de uma matriz $2 \times 2$, conforme mostrado no quadro 2 , onde o uso se caracteriza através da existência ou não de dados empíricos e de um modelo operacional.

O uso de simulação é bem antigo e anterior aos computadores. Becker (apud Kleiboer, 1997) indica que a primeira simulação foi um jogo de guerra, uma simulação de um fenômeno social feita em 1798 pelo exército prussiano como parte do treinamento de seus oficiais. Allen (1989) mostra que tal simulação, chamada "Krigspiel", foi desenvolvida e era utilizada como ferramenta de planejamento pelo exército alemão, antes da I Guerra Mundial. Tal metodologia foi adotada pelo exército dos EUA depois da II Guerra Mundial, mas só se popularizou efetivamente com o uso do computador, pela facilidade proporcionada por este na realização de cálculos complexos e das interações necessárias para resolver os modelos.

Quadro 2

Usos de simulação

\begin{tabular}{llcc}
\hline & \multicolumn{3}{c}{ EXISTEM DADOS EMPÍRICOS } \\
\hline \multirow{2}{*}{$\begin{array}{l}\text { EXISTE UM } \\
\text { MODELO }\end{array}$} & Sim & Sim & Não \\
\cline { 2 - 4 } & Não & Confirmatório & Projeção \\
\hline
\end{tabular}

Além do fato de que os recentes avanços em computação permitem programar nas chamadas "linguagens de alto nível", o mercado também disponibiliza pacotes de programação prontos que possibilitam elaborar modelos simples sem muito esforço. A capacidade computacional tem crescido num ritmo muito rápido e as ciências sociais têm sido lentas em acompanhar essa evolução, subutilizando os computadores. 


\section{Uso confirmatório}

Quando já se dispõe de um modelo operacional e dos dados empíricos, se utiliza a simulação para confirmar o modelo. Nessa categoria pode-se identificar o que Bruyne (1977, p. 108-109) chama de contexto da prova e contexto da descoberta.

O contexto da prova é aquele no qual se tenta provar ou refutar uma hipótese ou uma teoria. Assim, os dados estão sendo utilizados para testar o modelo. Muitos modelos são desenvolvidos com base num conjunto de dados, e quando submetidos a outro conjunto coletado sob condições diferentes podem ou não se mostrar capazes de predizer os resultados.

O contexto da descoberta é aquele no qual construímos as hipóteses. Isso acontece quando os dados refutam a teoria ou a hipótese, forçando a criação de novas hipóteses e a alteração do modelo. Tal fato pode decorrer de uma nova variável que precisa ser incorporada ou de uma função que não se comporta perfeitamente; ou seja, de uma disfunção.

Levando em conta que sempre existe um erro em cada modelo e que esses erros podem se somar conforme indica a teoria dos erros, deve-se levar em conta o princípio da parcimônia antes de se declarar um modelo, hipótese ou simulação inválidos e buscar uma nova solução. Um exemplo famoso do contexto da descoberta com simulações é descrito por Gleick (1991), quando o uso de simulações meteorológicas levou acidentalmente à descoberta do "efeito borboleta" em sistemas muito sensíveis a pequenas variações.

Uma variação do uso confirmatório é a de se alterar levemente os parâmetros da simulação para verificar sua sensibilidade e entender o quanto modelos ou hipóteses podem "tolerar". No contexto da prova, isso serve para mostrar a solidez de um modelo. Se um modelo é estável, mesmo com alterações, ele poderá futuramente ser submetido a um conjunto de dados novos e se mostrar apto a lidar com eles. Se o modelo é instável, isso revela um problema dentro do próprio modelo e pode gerar novas adaptações ou hipóteses, o que serve dentro do contexto da descoberta, isto é, o que alteraria o modelo dando-lhe estabilidade. Trata-se, portanto, quase de uma simulação dentro da simulação; ou seja, é utilizada a simulação para testar o modelo, o que pode ser especialmente útil em marketing, e economia, onde um pesquisador poderá testar até que tipo de parâmetro seu sistema é estável, se ele se perde com grandes variações ou se é insensível a pequenas variações. Isso pode ser comparado também ao que é feito com sistemas ainda experimentais, tais como aviões de teste ou navios recém-lançados, nos quais são testados os limites do sistema e a capacidade de recuperação do controle. Em aviação, chama-se "testar o envelope de vôo"(to test the flight envelop), e na Marinha, shake-down, que poderia ser traduzido como "amaciar a máquina". No sentido de Bertalanffy (1969), navios e aviões também são sistemas, tanto quanto programas de computador ou modelos científicos. Nesse sentido, seria possível afirmar que testar a estabilidade de um modelo, antes de expô-lo a críticas, é algo que faz bastante sentido e que tem um uso confirmatório do modelo ou de uma operacionalização deste, tal qual um programa de computador.

\section{Busca de um modelo}

Neste caso, dados empíricos estão disponíveis, mas não se tem ainda um modelo. Portanto, deverá ser criado um modelo inicial a ser aprimorado através de sucessivos testes.

Usualmente, uma idéia baseada na literatura teórica é um ponto de partida para se buscar um modelo funcionalista do objeto estudado.

Utilizando o princípio da parcimônia deve-se partir do mais simples para o mais complexo, sempre se embasando em teorias existentes e evitando variáveis não descritas na literatura, visando criar um modelo que seja sólido tanto do ponto de vista matemático-computacional quanto do teórico.

É sugerido que nenhuma linha de programação ou operação matemática seja tentada antes de se ter um modelo teórico feito com variáveis e setas relacionando-as através de interações. Certamente, isso é contra-intuitivo, uma vez que a tentação para escrever um código de computador ou modelos matemáticos é grande, mas limita 
a visão teórica do sistema e pode impossibilitar o avanço do modelo a partir do esboço inicial. Para criar um sistema como "a articulação das teorias em um todo" (BRUYNE et al., 1977), é preciso ver o todo não se concentrar apenas nas partes. Apenas com um modelo pronto se deve passar à programação de códigos ou planilhas e depois ao testes. Quando os testes indicarem novas necessidades, volta-se ao modelo inicial e somente depois disso, retorna-se ao programa.

É preciso ver os modelos iniciais como uma versão a ser aprimorada e não como uma versão definitiva. Nesse sentido, quando aprimorados os vários modelos, repete-se a superação que ocorre na ciência, pois, modelos mais simples vão sendo substituídos por mais complexos e completos à medida que o conhecimento avança. De certa forma, é o mesmo que ocorre no uso confirmatório no contexto da descoberta. Contudo, aqui, isso é feito de forma premeditada e intensiva, uma vez que existe um potencial ainda não explorado por muitos pesquisadores das ciências sociais, que é o de utilizar computadores e algoritmos para refinar modelos, testar hipóteses e comparar modelos alternativos. Embora isso possa ser feito de forma limitada com o uso de pacotes de simulação prontos, pacotes estatísticos e planilhas de cálculo, o maior potencial está no uso de softwares de programação puros, nos quais a liberdade é ainda maior. Sua dificuldade de uso esbarra no que Reibstein e Chussil (1999) explicam ser a dificuldade de encontrar quem entenda de programação e do objeto social ao mesmo tempo. Pode ser uma falha advinda do fato de que não existem cursos de programação em universidades de administração e em boa parte das de ciências sociais. Computadores e programação têm sido vistos como de uso predominante das ciências naturais, o que é apenas um preconceito, refletido no currículo das graduações e pós-graduações, não só no Brasil, mas também no exterior.

É quase um paradoxo que o avanço da tecnologia da informação e da telemática, tão citado e discutido pelos pesquisadores das ciências sociais e, em especial, pela administração, tenha sido relegado - tanto nos currículos escolares quanto no campo da pesquisa - a um segundo plano e que seja visto como algo "de fora". Se os órgãos de pesquisa em ciências sociais forem vistos como organizações em si, e o são, seriam considerados lentos na adoção das novas tecnologias, pois, na maioria das vezes, os microcomputadores são subaproveitados, uma vez que são usados meramente como máquinas de escrever sofisticadas.

\section{Projeção}

Neste caso já se tem um modelo validado por dados anteriores e o objetivo é fazer uma previsão sobre acontecimentos futuros, baseando-se nos dados do presente. Esse é o principal motivo para que se desenvolvam simulações dentro de organizações; ou seja, possibilitar a realização de previsões sobre o futuro que tendem a ser mais rigorosas do que outros métodos.

Reibstein e Chussil (1999) sugerem que as simulações sejam particularmente recomendáveis em situações complexas, na solução de problemas que não sejam familiares e quando o custo do erro for muito alto. Tais situações ocorrem em várias organizações, como naquelas que atuam em mercados sob forte pressão regulatória (telecomunicações e petróleo) ou onde certas práticas mercadológicas, foram proibidas, criando situações não-familiares (tabaco e, talvez, no futuro, bebidas alcoólicas e fast-food). Ocorrem igualmente em empresas com estratégia de liderança em custo, onde o erro é proibitivamente caro (varejo, commodities, agrobusiness e indústrias de base), e também em empresas em relação às quais a globalização transformou o mercado regional em global, aumentando muito a complexidade do negócio (automóveis, eletrônicos e mídia).

Existem ainda dois tipos de uso para as simulações de projeção: para treinamento ou para análise de inteligência. Quando a simulação é utilizada para treinamento, o objetivo é criar um cenário predefinido, no qual os alunos serão submetidos a um ambiente simulado, onde tomarão decisões baseadas em informações que receberem. Tais simulações podem ser uma poderosa ferramenta de ensino, pois estimulam os alunos a pensarem em todas as variáveis ao mesmo tempo e os coloca num ambiente de pressão similar ao da vida real. Curiosamente, essas simulações também costumam divertir os alunos, surgindo daí o neologismo "edutainment", isto é, educar ao mesmo tempo em que se entretêm.

Quando as simulações são empregadas para análise de inteligência, o propósito é o de criar cenários hipotéticos nos quais os competidores tomam um certo tipo de decisão ou no qual um evento externo às organizações 
competidoras altera o seu ambiente competitivo. Nesse caso, busca-se entender a dinâmica da mudança e geralmente se chega a um entendimento profundo do processo, além do que, são levantadas questões-chave do problema.

Contudo, tal entendimento (ou insight) costuma ocorrer sob o "efeito oráculo", isto é, não há uma resposta clara, mas, surge uma nova questão. Nesse ponto, mais uma vez, surge o contexto da descoberta; uma nova hipótese é criada de forma natural. Nesse sentido, é comum surgirem perguntas nunca feitas anteriormente durante simulações analíticas, permitindo pensar com antecedência ameaças futuras ou novas oportunidades.

Há também um grande risco quando se usam simulações, e que será chamado aqui "A falácia de Schielieffen". Quando os alemães, antes da I Guerra Mundial (portanto, antes dos computadores) desenvolveram uma simulação de guerra para prever os resultados de combates, o Kriegspiel (literalmente, "jogo de guerra"), eles o desenvolveram com dados validados no final do século XIX, utilizando-o para prever os resultados de uma invasão à França e recorrendo ao plano do general Schlieffen. Entretanto, o desenvolvimento de novos armamentos - especialmente, das metralhadoras - e a defesa obstinada dos belgas fizeram com que a simulação obtivesse resultados muito superiores aos conseguidos na prática (ALLEN, 1989).

Resumindo, a "falácia de Schlieffen" é acreditar totalmente no modelo, sem deixar nenhuma margem para a realidade. Pode-se dizer que essa falácia é o equivalente em simulações ao critério da "falseabilidade" de Popper; ou seja, o modelo pode sempre ser provado como estando errado. Assim, a projeção precisa ser validada pela realidade, a qual está sempre em constante mudança, surgindo mais uma vez o contexto da descoberta, dos modelos que precisam ser atualizados de forma sincronizada.

É comum uma organização ter duas versões de uma mesma simulação, uma para treinamento com cenários hipotéticos, e outra com os cenários verdadeiros ou prováveis, para análise de inteligência. Apesar dessas dificuldades Reibstein e Chussil (1999) sugerem que as simulações têm várias vantagens em seu uso para projeções, tais como: serem rigorosas, permitirem fazer "experiências" baratas e unificadoras e poderem ser feitas em sigilo. Em especial, as simulações permitem fazer "experimentos" virtuais que podem ser depois comparados com os resultados reais, de modo a aumentar a confiança num modelo ou refiná-lo.

\section{Projeção de comportamento}

Um tipo especial de projeção é a utilizada para analisar o comportamento dos indivíduos sob pressão, seja por causa de conflitos internos de um grupo com opiniões conflitantes, ou quando o indivíduo é forçado a escolher entre decisões que requerem relações de compromisso do tipo "curto prazo versus longo prazo" ou "alto risco/alto retorno versus baixo risco/baixo retorno". Essas situações são típicas de um ambiente organizacional e são parte central da tomada de decisão. Simulações que envolvem esse tipo de decisão podem ser utilizadas para analisar não o resultado do sistema, mas, sim, o comportamento de indivíduos que dela participam. Nesse caso, a simulação serve apenas como contexto para o pesquisador entender o comportamento dos indivíduos, pois, ela não é só uma metodologia, mas, também, uma ferramenta.

Embora num primeiro momento possa parecer estranho, é exatamente isso que é feito ao se responder às perguntas de um questionário. Coloca-se o respondente numa simulação sem computador e lhe é perguntado se prefere A ou o oposto da A. Esse tipo de pesquisa vem sendo feito há décadas, contribuindo para o entendimento de todo tipo de fenômeno social. A diferença numa simulação é que a pergunta não é feita diretamente, o que reduz a reflexividade da resposta; isto é, se reduz a probabilidade de que se responda algo falso apenas porque se quer agradar ao entrevistador ou por vergonha de responder a verdade. Numa simulação, é possível que o respondente nem saiba o que está sendo avaliado, muito menos que ele perceba que se trata de um teste. O lado negativo é que esse tipo de metodologia demora muito mais tempo para ser aplicada do que um questionário. 


\section{Exemplos interativos}

Para melhor ilustrar o observado anteriormente, serão explorados dois exemplos interativos, recorrendo-se a planilhas de Excell. Embora existam o Arena, o Stella, o Mathematica e o Statistica, as licenças desses softwares são caras. Por esse motivo, serão utilizados exemplos em Excell, que é mais facilmente encontrado nas diversas plataformas de trabalho e é mais difundido entre os usuários de PCs.

O primeiro exemplo é o de um uso confirmatório que leva a uma descoberta, baseado nos exemplos de Gleick (1991) e Priesmeyer (1993). Imagina-se um modelo no qual as vendas de um determinado produto, em termos de fatia de mercado (market share), são proporcionais às do período anterior, ainda que não linearmente. Em caso de vendas em demasia num determinado período, o mercado fica saturado e pode ocorrer um menor número de vendas no período seguinte.

A equação de venda num período $t+1$ é dado pela equação:

$$
X_{t+1}=r X_{t}\left(1-X_{t}\right)
$$

O arquivo de Excell "caos.xls", disponível em www.ebape.fgv.br/cadernosebape/caos.xls, mostra duas planilhas, uma contendo os cálculos das fatias de mercado ao longo de vários períodos, utilizando a fórmula anterior, e outra com o gráfico referente. A célula marcada de azul claro contém o valor de "r", e inicialmente aparece o valor de 2,5. Pede-se ao leitor que altere esse valor de $0,1 \mathrm{em} 0,1$; isto é, coloque 2,6, depois $2,7 \mathrm{e}$ assim por diante, observando o que ocorre tanto com os números quanto com o gráfico, até que " $r$ " chegue a 4,1 .

Inicialmente em 2,5 o comportamento do sistema é simples, ficando mais complexo a medida que "r" sobe, até que a partir de 3 fica cada vez mais complexo, ou "caótico". Em 4,1 o comportamento se torna instável demais e a fatia de mercado supera $100 \%$ para depois ir abaixo de $0 \%$. Isto é, o sistema não mais funciona e a equação não é mais capaz de predizer o comportamento do mercado.

Se o leitor continuar a elevar o valor de "r", verá comportamentos cada vez mais erráticos até que, quando "r" é igual a 5, surge um comportamento bem comportado, que volta a desaparecer com " $r$ " igual a 5,1 ou 4,9. Isso representa uma "ilha de estabilidade".

Esse exemplo levou à descoberta de modelos aparentemente bem comportados que podem se tornar erráticos, complexos ou "caóticos", dependendo da variação de um único componente da equação. Um uso confirmatório pode levar a uma descoberta; nesse caso, uma das descobertas que levaram à teoria da complexidade, também chamada de teoria do caos ou teoria do caos determinístico.

O segundo exemplo descreve o comportamento de preços utilizando o modelo de Cobweb para a oferta e a demanda. Nesse modelo, a oferta de um determinado produto é baseada nos preços vigentes num período anterior e a demanda atual é igual a essa oferta. Entretanto, isso regula o preço de acordo com as seguintes equações:

$$
\begin{aligned}
& D_{t}=a-b P_{t} \\
& O_{t}=c+d P_{t-1} \\
& D_{t}=O_{t}
\end{aligned}
$$

Esse tipo de comportamento ocorre quando existe um "retardo" no ajuste da oferta em relação aos preços, o que é verdadeiro para mercados nos quais a infra-estrutura necessária para atender à demanda leva tempo para ser construída. Esse exemplo permite ver o uso de uma projeção de preços a partir de um conjunto de dados previamente levantados. Isso significa que já se tem um modelo e que esse modelo está ajustado de acordo com dados anteriores, e que, agora, pretende-se utilizar o modelo para prever os preços futuros. 
O arquivo de Excell "cobweb.xls", disponível em www.ebape.fgv.br/cadernosebape/cobweb.xls, contém cinco planilhas. A primeira, chamada de "modelo", contém as fórmulas e os cálculos baseados em dois conjuntos de valores para as variáveis "a", "b", "c", "d" e "P0". A célula em azul claro mostra o número do conjunto que está sendo usado. Será pedido ao leitor que o altere de "1" para "2" e que veja quais as conseqüências nos gráficos de "preços" e "espirais". As duas planilhas restantes contêm cálculos necessários para a montagem do gráfico de "espirais".

Deve ser observado que com o conjunto " 1 " os preços tendem a se estabilizar, cada vez mais próximos do ponto de equilíbrio, enquanto as espirais convergem para o ponto de equilíbrio da oferta e da demanda, como previsto por modelos econômicos convencionais. Porém, com o conjunto " 2 " os preços divergem do preço inicial e as espirais migram "para fora", não havendo equilíbrio. Dessa forma, é possível prever se um determinado produto irá atingir ou não um ponto de convergência natural. Assim, será possível a um gestor privado determinar se vale a pena ou não entrar nesse mercado, e a um gestor público, avaliar se o mercado necessita de regulação ou não.

É importante salientar que uma metodologia estatística não será capaz de prever os preços futuros se o produto tiver um comportamento similar ao gerado pelo conjunto " 2 ", e que terá dificuldades de prever o comportamento dos preços com o conjunto " 1 ". O valor de $\mathrm{R}^{2}$, localizada logo abaixo da legenda da planilha de preços, através de uma curva de tendência linear é colocado no arquivo para denotar o baixo poder explicativo de uma metodologia estatística, nesse caso.

Muitos outros exemplos seriam possíveis, mas essas duas situações demonstram como uma ferramenta simples como o Excell pode ser utilizada em diversos contextos de pesquisa. Outras ferramentas bem mais capazes do que o Excell podem ser encontradas no mercado, embora sejam mais caras e menos difundidas.

\section{Conclusão}

É possível integrar os três tipos de uso num ciclo de pesquisa. Primeiro, são obtidos os dados para criar uma simulação, com ela são feitas projeções baseadas nos dados atuais e, depois, o modelo é confirmado (ou não). Com os novos dados é possível fazer novas projeções, a serem confirmadas ou não, de modo que o aperfeiçoamento desse mesmo modelo é contínuo.

Essa é uma alternativa barata à experimentação, tanto em ciências sociais como particularmente na administração. A experimentação, mesmo quando possível, é quase sempre muito cara, especialmente, nas áreas de marketing e estratégia, de contornos muito amplos. Embora a simulação não substitua a experimentação, pode criar modelos sofisticados a partir de experimentos simples que comprovem as partes do modelo.

A principal vantagem das simulações é permitir antever problemas de primeira ordem e gerar perguntas que não eram formuladas antes. Entretanto, também correm o risco de se tornarem uma espécie de "figura de Roschard", quando confirmam as expectativas dos gerentes, uma vez que mostram o que estes querem ver.

Apesar dessas vantagens, as simulações têm sido relegadas a um segundo plano em relação aos estudos de caso e às amostragens, em face da dificuldade de praticar a experimentação. Embora este estudo não apresente evidências empíricas para afirmar as razões dessa negligência, fica lançada, aqui, a hipótese de que esta resulte do currículo das graduações e pós-graduações na área de administração. Também seria conseqüência do fato de que os cursos de ciências sociais geralmente carecem de matérias que ensinem o uso de linguagens de programação.

Finalmente, as simulações devem ser vistas como uma ferramenta importante de investigação do mundo real, não como "a verdade". Aqui se reproduz o drama da ciência como um todo, em que há a tendência de que os modelos sejam vistos como "a verdade" até que a realidade se mostre de uma forma diferente, transformando em falsos (ou falseando) os modelos anteriores. Nas simulações, como na ciência em geral, deve-se buscar uma superação, processo este que se repete continuamente. 


\section{Referências bibliográficas}

ALLEN, T. B. War games. New York: Berkley Books, 1989.

BERTALANFFY, L. V. General system theory. New York: George Braziller, 1969.

BURREL, G.; MORGAN, G. Sociological paradigms and organizational analysis. London: Heineman, 1982.

BRUYNE, P. et al. Dinâmica da pesquisa em ciências sociais. Rio de Janeiro: Francisco Alves, 1977.

DAY, G. S.; REIBSTEIN, D. J . A dinâmica da estratégia competitiva. Rio de Janeiro: Campus, 1999.

DOYLE, J. Bounded rationality. In: WILSON, R. A.; KEIL, F. C. The MIT Encyclopedia of the cognitive sciences. Cambrige: The MIT PresS, 1999.

GLEICK, J. Caos: a criação de uma nova ciência. Rio de Janeiro: Campus, 1991.

HEIJDEN, K. V. D. Scenarios: the art of strategic conversation. New York: John Wiley \& sons,1996.

KLEIBOER, M. Simulation methodology for crisis management support. Journal of Contingencies and Crisis Management, v.5, n.4, p.198206, Dec. 1997.

ORDESHOOK, P. C. Game theory and political theory. New York: Cambridge University Press, 1986.

OSBORNE, M. J.; RUBINSTEIN, A. A course in game theory. Cambridge: The MIT Press, 1994.

PRIESM EYER, R. H. Organizations and chaos: defining the methods of nonlinear management. Westport: Quorum Books, 1993.

REIBSTEIN, D. J.; CHUSSIL, M. J. Primeiro a lição, depois o teste: usando simulações para analisar e desenvolver estratégias competitivas In: DAY, G. S.; REIBSTEIN, D. J . A dinâmica da estratégia competitiva. Rio de Janeiro: Campus, 1999.

RINGLAND, G.; Scenario planning: managing for the future. New York: J ohn Wiley \& sons, 1998.

SCHWARTZ, P. A arte da visão de longo prazo. São Paulo: Best Seller, 2000.

STERMAN, J. D. System dynamics modeling: tools for learning in a complex world. California Management Review, v.43, n.4, 2001.

WILSON, R. A.; KEIL, F. C. The MIT Encyclopedia of the cognitive sciences. Cambridge: The MIT Press, 1999.

ZADEH, L. A. Fuzzy logic. In: WILSON, R. A.; KEIL, F. C. The MIT Encyclopedia of the cognitive sciences. Cambridge: The MIT Press, 1999. 